\title{
Insights on Usability and User Feedback for an Assistive Robotic Health Companion with Adaptive Linguistic Style
}

\author{
Hannes Ritschel, Kathrin Janowski, Andreas Seiderer, Stefan Wagner and Elisabeth André \\ Human-Centered Multimedia, Augsburg University \\ Augsburg, Germany \\ \{ritschel,janowski,seiderer,andre\}@hcm-lab.de
}

\begin{abstract}
Socially assistive robots help the human in everyday tasks. In particular, they can also provide assistance with regard to health and wellbeing, which is an important opportunity to support the independence of the elderly. Since spoken language often carries the essential information, the robot's verbal communication style is of special interest. In order to explore individual user's preferences with regard to the robot's linguistic style we built an autonomous, assistive health companion. It is operated with a custom hardware control panel and offers games, jokes, health-related recommendations, applications for information retrieval and communication. This paper reports on insights from the in-situ user study with regard to the system's usability and participants' feedback.
\end{abstract}

\section{CCS CONCEPTS}

- Computing methodologies $\rightarrow$ Intelligent agents; Reinforcement learning; $\bullet$ Human-centered computing $\rightarrow$ Haptic devices;

\section{KEYWORDS}

social robots; assistive companions; linguistic style; adaptation

\section{ACM Reference Format:}

Hannes Ritschel, Kathrin Janowski, Andreas Seiderer, Stefan Wagner and Elisabeth André. 2019. Insights on Usability and User Feedback for an Assistive Robotic Health Companion with Adaptive Linguistic Style. In The 12th PErvasive Technologies Related to Assistive Environments Conference (PETRA '19), June 5-7, 2019, Rhodes, Greece. ACM, New York, NY, USA, 2 pages. https://doi.org/10.1145/3316782.3322737

\section{INTRODUCTION}

Robots will be part of our future to assist, facilitate and enrich our everyday lives. Entertainment, information retrieval and communication will be important tasks. Especially with regard to health and wellbeing, robots will also act as assistant and advisor. Even today, the commercial Pillo robot integrates such functionality: its main tasks are reminding family members of taking pills, as well as answering questions regarding the nutritional intake of food. Further examples of similar consumer products are fibo, Buddy, Mabu, ElliQ, Lynx and Moorebot.

(C) Authors | ACM 2019. This is the author's version of the work. It is posted here for your personal use. Not for redistribution. The definitive Version of Record can be found at https://doi.org/10.1145/3316782.3322737
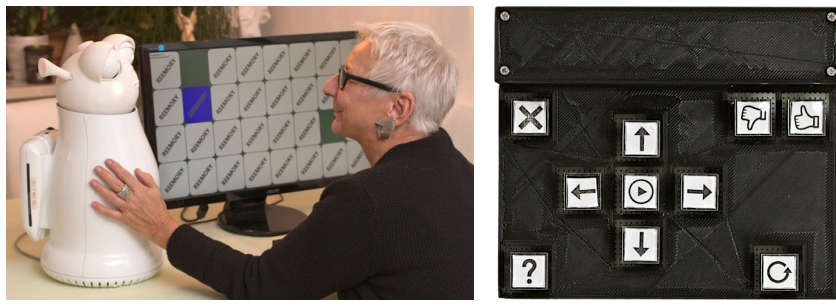

Figure 1: Female study participant and control panel with physical buttons to interact with the robotic companion.

In order to support the independence of the elderly population, robotic companions have long been subject of research. For example, Sidner et al. [8] developed a social companion agent for the elderly which offers various activities for entertainment, communication as well as exercises. Interaction with the robot is achieved primarily with a touch screen. Schroeter et al. [7] presented a socially assistive robot companion for older people suffering from mild cognitive impairment. It reminds of appointments and activities, makes video calls, includes a cognitive stimulation game and is linked to smart home technologies. Especially with regard to health and wellbeing attempts are made for example to support everyday life by providing information about the nutritional value of drinks based on the human's drink consumption [5]. The support of exercises is the main task in robot-assisted training [9] with the goal to increase enjoyment and intrinsic motivation. In this context, research also addresses the elderly population, such as with arm, finger or cognitive training [2].

\section{AN ASSISTIVE ROBOTIC COMPANION}

Inspired by [8] and [7] we built an assistive companion [6] with adaptive linguistic style in order to investigate the role and impact of the robot's communicative behavior. It is equipped with games, jokes, health-related recommendations, information retrieval for news, weather forecast, appointments and contacts as well as email and chat communication. Depending on the current task it explores different politeness strategies or types of expressed persona during runtime. It tailors its linguistic style to the individual user's preferences based on their explicit evaluative feedback.

The interaction with the robotic companion is realized completely with the help of a custom-built, 3D printed control panel (see Figure 1). It was implemented with physical buttons due to issues reported in the literature with respect to touch-based interaction in the context of the elderly domain. Ten physical buttons with integrated LED lights allow giving explicit positive or negative feedback for the linguistic adaptation process, to navigate through 
Table 1: Questionnaire results.

\begin{tabular}{ll||cl|cl}
\hline \multicolumn{2}{l||}{ Questionnaire } & \multicolumn{2}{c|}{ female subject } & \multicolumn{2}{c}{ male subject } \\
\hline \hline TA-EG & Enthusiasm & 3.8 & of 5 & 3.2 & of 5 \\
& Competence & 4.0 & of 5 & 4.3 & of 5 \\
& Positive Effects & 4.6 & of 5 & 4.0 & of 5 \\
& Negative Effects & 1.8 & of 5 & 2.4 & of 5 \\
\hline SUS & & 100 & of 100 & 90 & of 100 \\
\hline AttrakDiff & PQ & 6.0 & of 7 & 6.6 & of 7 \\
& HQ-I & 6.1 & of 7 & 5.7 & of 7 \\
& HQ-S & 5.0 & of 7 & 6.4 & of 7 \\
& ATT & 6.6 & of 7 & 6.3 & of 7 \\
\hline
\end{tabular}

the menu and choose selections, to start an application or confirm selections, to stop the robot's text output or quit an application, to repeat the robot's last utterance and to provide help.

\section{USABILITY}

At the time of this writing two test persons participated in the ongoing in-situ study. Both the female user (aged 64) and the male user (aged 61) were German native speakers. The autonomous robot was placed in their homes (see Figure 1) for one week, where they were free to interact with it as often as they wanted.

At the beginning of the study, the participants were asked to fill in the TA-EG questionnaire [4] about their attitude towards electronic devices. It measures four aspects: Enthusiasm, Competence, Positive Effects of Technology and Negative Effects of Technology. As shown in Table 1, both participants scored rather high on the aspects competence and positive consequences. On the enthusiasm aspect, the woman scored rather high whereas the man's attitude was closer to the neutral value of 3.0. As for the aspect negative consequences, both were below neutral. The results indicate a positive, optimistic opinion about electronic devices and confidence in the ability to handle them. This is not overly common among this demographic, so their affinity with modern technology was probably the reason why both test persons agreed to participate in the study.

To evaluate the custom-made control panel, we asked the participants to fill in the System Usability Scale [1] after the study. According to their answers, the interface achieved very high scores of 100 respectively 90 out of 100 , so evidently both perceived it as straightforward to use and did not experience problems. This is in line with their perceived competence in handling electronic devices as previously measured by the TA-EG questionnaire.

\section{PARTICIPANTS' FEEDBACK}

After the study, we interviewed the participants to gain a deeper understanding of their experience with the system. To measure the overall perception of the system, we asked the participants to rate it on the AttrakDiff [3] questionnaire (see Table 1). The woman's opinion was positive with regard to Attractivity (ATT), Pragmatic Quality (PQ) and Hedonic Quality - Identity (HQ-I). On the Hedonic Quality - Stimulation (HQ-S) subscale, the system achieved a lower score, but still above the neutral value of 4.0. The man rated the system rather positive on all subscales, the lowest rating being on the HQ-I subscale (5.7 of 7). Overall, these results are in line with the TA-EG questionnaire which had revealed both users' high affinity with modern technology.

Both participants mentioned that they would also like to use spoken language to interact with the robot, e.g. to reply to received messages. The female person pointed out that this would be a privacy concern and that embedding cloud services would not be an option at all. She would not have participated if cloud-based speech recognition was embedded in the robot. Offline speech recognition would be essential which handles the user's data transparently. Furthermore, she pointed out that she would like to cancel longwinded speech output earlier. The male participant noted that the robot switched between the informal and formal pronouns ("Du" and "Sie" in German), which could occur due to the robot's different politeness strategies. This shows that in languages with these kinds of differences special care should be taken when a learning system explores different degrees of formality.

\section{DISCUSSION}

The robot was perceived as attractive and easy to use, which is in line with both users' preexisting affinity with technology. Both participants were able to interact with the system as intended and successfully used the control panel to give feedback to the adaptation process. Since participants' feedback shows that privacy is still a concern, autonomous technologies, such as assistive companions, should be built in a transparent and responsible manner. Moreover, the experiment indicates that evaluating a robot's language is hard due to fact that efficiency may be more important than politeness.

\section{ACKNOWLEDGMENTS}

This research was funded by the Bavarian State Ministry for Education, Science and the Arts (StMWFK) as part of the ForGenderCare research association.

\section{REFERENCES}

[1] John Brooke. 1996. SUS - A quick and dirty usability scale. Usability evaluation in industry 189, 194 (1996), 4-7.

[2] Juan Fasola and Maja J Matarić. 2013. A Socially Assistive Robot Exercise Coach for the Elderly. 7. Hum.-Robot Interact. 2, 2 (jun 2013), 3-32.

[3] Marc Hassenzahl, Michael Burmester, and Franz Koller. 2003. AttrakDiff: Ein Fragebogen zur Messung wahrgenommener hedonischer und pragmatischer Qualität. In Mensch \& Computer 2003. Vol. 57. Vieweg+Teubner Verlag, Wiesbaden, 187-196.

[4] Katja Karrer, Charlotte Glaser, Caroline Clemens, and Carmen Bruder. 2009. Technikaffinität erfassen - der Fragebogen TA-EG. ZMMS Spektrum 29 (09 2009).

[5] Hannes Ritschel, Andreas Seiderer, Kathrin Janowski, Ilhan Aslan, and Elisabeth André. 2018. Drink-O-Mender: An Adaptive Robotic Drink Adviser. In Proceedings of the 3rd International Workshop on Multisensory Approaches to Human-Food Interaction (MHFI'18). ACM, New York, NY, USA, Article 3, 8 pages.

[6] Hannes Ritschel, Andreas Seiderer, Kathrin Janowski, Stefan Wagner, and Elisabeth André. 2019. Adaptive Linguistic Style for an Assistive Robotic Health Companion Based on Explicit Human Feedback. In Proceedings of the 12th PErvasive Technologies Related to Assistive Environments Conference. ACM.

[7] Ch. Schroeter, Steffen Müller, Michael Volkhardt, Erik Einhorn, Claire A. G. J. Huijnen, Herjan van den Heuvel, Andreas van Berlo, Andreas Bley, and HorstMichael Gross. 2013. Realization and user evaluation of a companion robot for people with mild cognitive impairments. In 2013 IEEE International Conference on Robotics and Automation, Karlsruhe, Germany, May 6-10, 2013. IEEE, 1153-1159.

[8] Candace L. Sidner, Timothy W. Bickmore, Bahador Nooraie, Charles Rich, Lazlo Ring, Mahni Shayganfar, and Laura Vardoulakis. 2018. Creating New Technologies for Companionable Agents to Support Isolated Older Adults. TiiS 8, 3 (2018), $17: 1-17: 27$.

[9] Konstantinos Tsiakas, Maria Kyrarini, Vangelis Karkaletsis, Fillia Makedon, and Oliver Korn. 2018. A Taxonomy in Robot-Assisted Training: Current Trends, Needs and Challenges. Technologies 6, 4 (2018). 